\title{
Anastrozole as aromatase inhibitor - new approaches to breast cancer treatment in postmenopausal women
}

\author{
Izabela Piotrowska, Magdalena Piotrowska
}

Chair of Drug Technology and Biochemistry, Faculty of Chemistry, Gdańsk University of Technology, Poland

Introduction. Anastrozole is a chemotherapeutic drug used to treat breast cancer in postmenopausal women. The condition for success of this type of treatment is the presence of estrogen receptors in cancer cells. Anastrozole belongs to strong-acting, nonsteroidal inhibitors of aromatase, which is responsible for androgen-estrogen conversion process. The metabolism of this drug is based on biotransformation both in phase I and II. Initially it metabolizes in the liver with cytochrome P450 isoenzymes, followed by a glucuronidation reaction catalyzed by the UGT1A4 enzyme.

Material and method. In order to minimize the side effects, new therapeutic approaches are sought to increase the success of the therapy.

Conclusions. Biodegradable nano-tools that deliver anastrozole to the target site with an increase in its half-life to 144 hours are a promising method. A similar effect can be achieved by using a transdermal anastrozole administration system, which is a completely non-invasive method of treatment.

NOWOTWORY J Oncol 2019; 69, 1: 26-35

Key words: anastrozole, aromatase, P450 cytochrome, nanotechnology, transdermal system

\section{Introduction}

Breast cancer depends on many factors. One of the important elements of risk is the age of the patient - the risk of falling ill increases with age (up to a certain age limit). The vast majority of breast cancers concern women in postmenopausal age. Paradoxically, breast tumors in postmenopausal women show favorable prognosis associated with increased expression of steroid sex hormones receptors. Estrogens, belonging to the group of steroid sex hormones, support the growth and survival of both normal and cancer cells. This is made possible by the binding and activation of the estrogen receptor (ER). Its presence in neoplastic cells, i.e. the type of neoplasm defined as ER+, is found in most breast neoplasms. This indicates a good prognosis of patient survival with the use of pharmacological treatment with anti-estrogens. An estrogen receptor activated by $17-\beta$-estradiol works on the basis of a transcription factor that activates the expression of specific genes. They are mainly responsible for cell growth, prolonged survival and proliferation of cancer cells. Since in most cases the incidence of breast cancer depends on the level of hormones, it can be considered that hormone therapy can be an effective method of complementary treatment. There are three ways in which estrogen-dependent processes, important for the development and progression of most breast cancers, can be inhibited. The first is impacting the estrogen binding with the ER and/or the elements of the gene promoter it codes. An example of such action are selective ER modulators, i.e. tamoxifen and raloxifene. The second method is to reduce or eliminate the expression of ER, which is shown by the fulvestrant - a selective regulator decreasing ER. However, the most effective way of therapy is to reduce the amount of estrogen by inhibiting its production. Such pattern of action is demonstrated by aromatase inhibitors (AI), which include anastrozole (Fig. 1). The factor determining their effectiveness is the sensitivity of cancer cells to hormones, i.e. the presence of estrogen receptors in them $[1,2]$. 


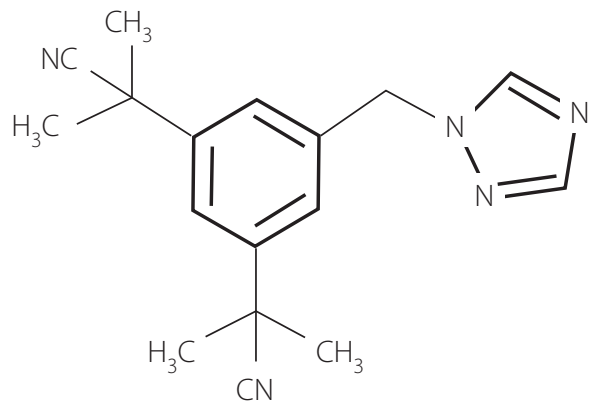

Figure 1. Chemical structure of anastrozole

\section{Importance of anastrozole in clinical practice}

Based on the available published data, anastrozole can be considered the most selective third generation aromatase inhibitor in clinical conditions. It does not reveal a weakened response to adrenocorticotropic hormone (ACTH) stimulation even after 10 administrations of normal clinical dose of anastrozole for 4 weeks, which suggests that anastrozole therapy does not affect adrenal cortex hormone synthesis [3].

Anastrozole was the first aromatase inhibitor to show significant survival benefits compared to the standard second-line drug: megestrol acetate. Two phase III clinical trials after 6 months have shown that anastrozole at $1 \mathrm{mg}$ daily is as effective as megestrol acetate, but its use did not affect the significant weight gain observed when megestrol acetate was administered. This analysis also showed a higher median survival rate for the benefit of anastrozole (22.5 months in the megestrol acetate group vs 26.9 months in the anastrozole group). No additional benefit was observed after increasing the dose to $10 \mathrm{mg}$, thus supporting the choice of $1 \mathrm{mg}$ as a clinical dose. Anastrozole was subsequently approved by the US Food and Drug Administration for use in the second line of treatment for postmenopausal women with advanced breast cancer [4].

In 2000, two clinical programs were developed to compare the efficacy and tolerance of anastrozole and tamoxifen in women with advanced breast cancer. Programs: "TARGET" (Tamoxifen or Arimidex ${ }^{\text {TM }}$ Randomized Group Efficiency and Tolerability) and "North American" included patients with newly diagnosed disease or cancer progression following previous treatment. They have been randomly assigned to a dose of $1 \mathrm{mg}$ anastrozole daily or $20 \mathrm{mg}$ of tamoxifen daily. The results of the "North American" study showed a clear superiority of anastrozole over tamoxifen in terms of disease progression time (TTP $=11.1$ months for anastrozole and 5.6 months for tamoxifen). A 44\% longer survival time without disease was also shown for patients receiving anastrozole compared to patients treated with tamoxifen. The TARGET study also confirmed beneficial effects of anastrozole compared to tamoxifen. After obtaining the results of these studies, anastrozole has been approved in many countries as a first-line treatment for advanced breast cancer in postmenopausal women [3].
It also meets the necessary criteria to be considered effective as a complementary treatment in postmenopausal women. These criteria include: better efficacy if compared with the existing adjuvant agents, better tolerance and easy and convenient dosage [3].

The latest FACE (Final Anastrozole Clinical Evaluation) study was designed to evaluate the efficacy and safety of adjuvant letrozole (LET) compared to anastrozole (ANA) in postmenopausal breast cancer patients with hormone receptor-positive breast cancers. The total number of patients was 4170, which were randomly assigned to the group receiving letrozole $(n=$ 2076) or anastrozole $(n=2094)$. For 5 years they were administered a standard clinical dose of anastrozole (1 mg daily) or letrozole (2.5 mg daily). The primary endpoint of the study was a 5 -year disease-free survival (DFS). The key secondary endpoints were security and overall survival (OS). The five-year estimated DFS ratio was $84.9 \%$ for LET and $82.9 \%$ for ANA, while the OS ratio was $89.9 \%$ (LET) and $89.2 \%$ (ANA). The main reasons of treatment discontinuation in the letrozole arm compared to the anastrozole arm were side effects (15.1\% vs 14.3\%) and disease progression (9.5\% vs $10.4 \%)$. The safety profiles were similar in both treatment arms. The most common side effects of letrozole compared to anastrozole were: joint pain (48.2\% vs $47.9 \%$ ), hot flushes (32.5\% vs $32.3 \%$ ) and fatigue (16.8\% vs 16.6\%). Suspected adverse reactions leading to discontinuation of treatment were reported in $14.0 \%$ of LET patients and $12.9 \%$ of ANA patients respectively [5].

\section{P450 as estrogen metabolizing enzymes}

Cytochrome P450 isoenzymes play an important role in the biosynthesis of bioactive compounds such as sterols, steroid hormones, eicosanoids and vitamins. Moreover, they participate in the detoxification of xenobiotics, but one of their most important functions is drug metabolism [6, 7]. Isoenzymes are also involved in the metabolism of estrogens. The addition of hydroxyl group at C-2 position is the main metabolic pathway of estradiol in the liver mediated by P450 1A2, 2C8, 2C9 and $3 \mathrm{~A} 4$. Estrogens undergo biotransformation as a result of 2/4-hydroxylation reaction, catalyzed mainly by $\mathrm{P} 4503 \mathrm{~A} 4$ at the ortho position to the 3-phenol 2/4-hydroxyestrogen form. These metabolites are then converted to 2/4-methoxy-estrogens with catechol-O-methyltransferase (COMT). P450 enzymes also catalyze the hydroxylation reaction at position 16-a, which results in the formation of 6a-hydroxyestrone (Fig. 2) [8, 9].

Aromatase (P450 19) is an enzymatic complex, responsible for catalyzing the biosynthesis of estrogens from androgens, associated with endoplasmic reticulum in cells. This complex is coded by a single CYP19 gene located in the human chromosome 15 at position 21.2. Aromatase is also called estrogen synthetase, which consists of two functional proteins. The first one is the P450 cytochrome, called monooxygenase. The second is a non-specific flavoprotein microsomal reductase, also known as NADPH cytochrome P450 reductase, used as 
a cofactor, nicotinamide adenine dinucleotide. In addition, aromatase is highly expressed in Graafian follicles and placenta. In postmenopausal women, most estrogens are produced by adrenal cortex. Aromatase in low concentrations also occurs in breast tissue, adipose tissue, brain, liver and muscles [10-12]

Androgens such as testosterone, dihydrotestosterone and androstenedione are converted to estrogens using cytochrome P450 aromatase (Fig. 3). Estrogens are formed as a result of the loss of the C-19 lateral methyl group in androgens and the formation of an aromatic ring. Estrone (E1) is synthesized from androstenedione and estradiol (E2) from testosterone. The aromatization reaction, i.e. the formation of an aromatic ring, is directly catalyzed with the participation of P450 19.

The most important step in this process is the elimination of the C-19 group, which enables the aromatization of the $A$ ring in the molecule. This process takes place after three oxidation reactions beginning with hydroxylation of the methyl group at C-19 position. The last stage is a reaction eliminating the rest of C-19 as formic acid. The product created by the keto-enol tautomerism strives to aromatize the A ring. To transform the androstenedione molecule to the esterone molecule, three NADPH molecules are therefore needed, together with oxygen, to form water and nicotinamide adenine dinucleotide phosphate (NADP+) during the reaction. In parallel to the aromatization, a hydroxylation reaction with $\mathrm{NADPH}+\mathrm{H}+[12$, 13] takes place.

\section{Anastrozole as active substance}

Anastrozole (2-[3-(2-cyanopropan-2-yl)-5-(1,2,4-triazol-1-ylmethyl)phenyl]-2-methylpropanenitrile) contains in its structure an aromatic ring with three substituents, which consist of methyl, nitrile and triazole groups. It is therefore a chiral derivative of triazole catalyzed by P450 cytochrome, which results in classifying it as a powerful inhibitor and inductor of drug metabolism. Moreover, lipophilic compounds containing heterocyclic nitrogen substituents in their structure inhibit monooxygenase activity in the liver, with variable affinity of binding to microsomal membranes depending on heterocyclic substitution position. More lipophilic compounds penetrate the membranes more easily and are therefore metabolized mainly in the liver by microsomal enzymes. Lipophilicity of anastrozole expressed as $\log \mathrm{P}_{\mathrm{o} / \mathrm{w}}$ ratio is 1.58 - which means that it is a moderately lipophilic compound $[14,15]$.

The growth and development of breast cancer depends on the level of estrogen. Therefore, aromatase inhibitors, which are responsible for their biosynthesis, are often used to treat this type of cancer. Reducing estrogen levels is the main objective of hormone therapy for the treatment of breast cancer with positive

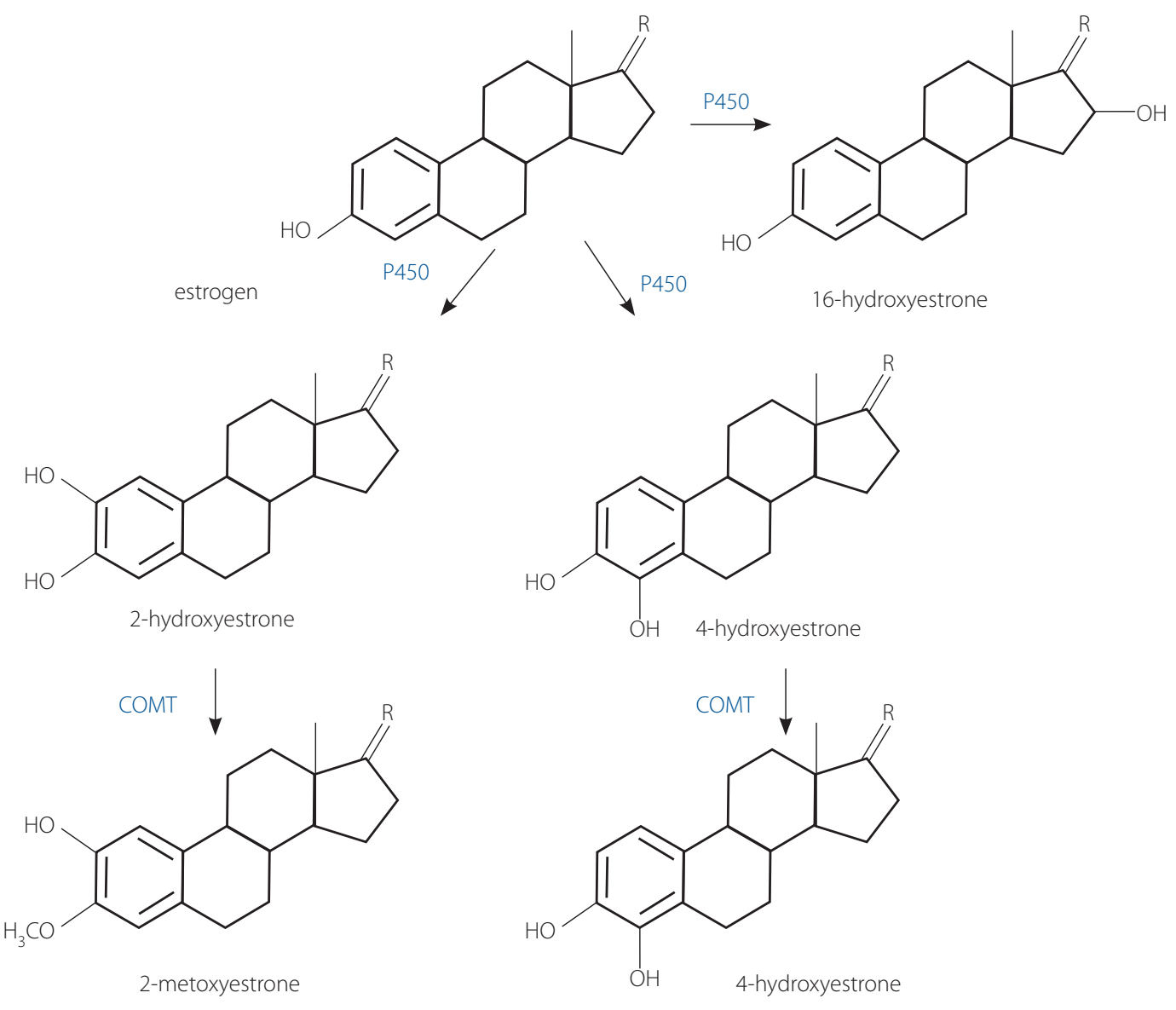

Figure 2. Estrogen metabolism in the presence of P450 cytochrome enzymes 


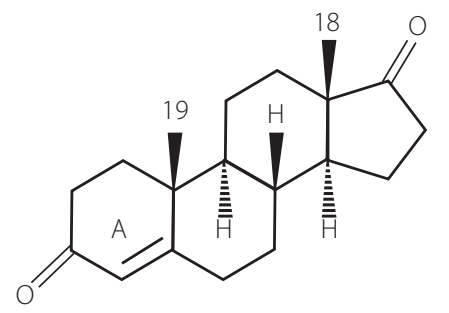

$\stackrel{\mathrm{O}_{2} \mathrm{NADPH}}{\longrightarrow}$ androstenedione $\left(\mathrm{C}_{19}\right)$

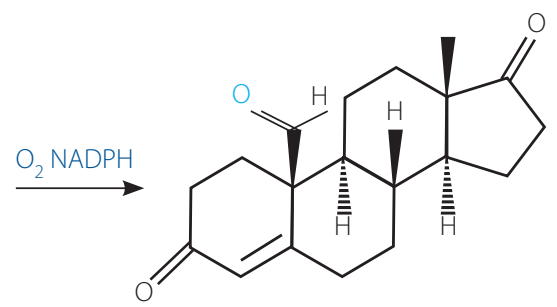

CYP19-Fe-OOH
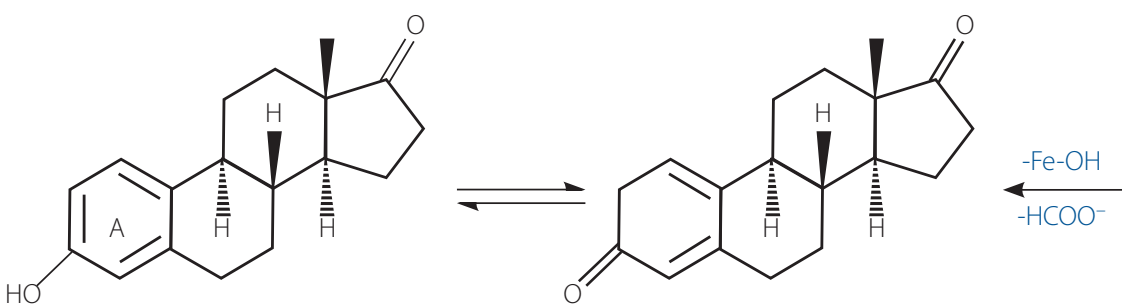

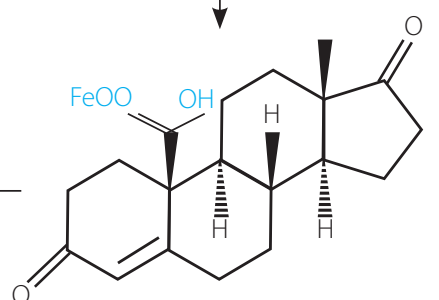

estrone $\left(C_{18}\right)$

estrone $\left(C_{18}\right)$

Figure 3. A reaction catalyzed by aromatase. Loss of lateral C-19 group with formation of an aromatic ring

hormone receptor effects. Estrogen reduction can be achieved in two ways: by blocking the estrogen receptor or by inhibiting estrogen biosynthesis with aromatase. The mechanism of action of anastrozole should lead to inhibition of estrogen biosynthesis. Clinical trials confirm the efficacy of Al interaction with estrogen receptor in metastatic breast cancer treatment in postmenopausal women. In addition, a number of studies have confirmed that Al are more effective in postmenopausal women than tamoxifen commonly used in adjuvant therapy [16].

Al are divided into two groups in terms of inhibition of the aromatization process. The first group, which includes exemestane, is irreversibly linked to aromatase and causes its permanent inactivation. The second group, which includes anastrozole, shows reversible binding to P45019, competing with endogenous ligands to the active enzyme site. This is done by creating a reversible binding between the $\mathrm{N}-4$ nitrogen group in the triazole ring and the iron atom in the active centre of the P450 cytochrome (Fig. 4). Anastrozole is therefore a competitive, nonsteroid P45019 inhibitor that selectively inhibits the conversion of androgens to estrogens. As result, the concentration of estrone, estradiol and estrone sulphate circulating in the serum decreases. Inhibition of aromatase by anastrozole consists mainly in inhibition of P450 1A2, 2C8, 2C9, 3A4 isoenzymes, which are involved in estrogen metabolism [8].

\section{Metabolism of anastrozole}

The effect of a drug in view of biological purposes depends on its specific structural modification. This biotransformation is most commonly carried out in the liver and its aim is to convert lipophilic substances into more hydrophilic metabolites that will be excreted. After being absorbed into the cell, the active substance is metabolized, i.e. which is divided into two main phases. In the first phase there is an increase in the hydrophilicity of drugs, which facilitates and enables their excretion. In this phase, oxidation, reduction, elimination and hydrolysis reactions occur. Introducing a functional group into a drug molecule enables its reaction and then interaction in phase II reactions. Oxidation processes are the most important biotransformation pathway. They are catalyzed, among others, by monoxygenases, which build into xenobiotics one oxygen atom coming from an oxygen molecule, while the other one is reduced and a water molecule is formed. The most important for this phase of metabolism are the enzymes from the family of P450 cytochrome, which are catalysts of many biochemical transformations [12].

Anastrozole is metabolized in the liver is mainly due to hydroxylation - catalyzed by P450 3A4 enzyme and to a lesser extent by P450 3A5 enzyme - with hydroxy-anastrozole formation. The second important transformation of the drug is $\mathrm{N}$-dealkylation, which results in the formation of metabolites: triazole and 3,5-bis-(2-methylpropiononitrile)-benzoic acid (Fig. 5). The main anastrozole metabolites identified in urine and plasma after phase I of elimination are triazole and hydroxy-anastrozole. Triazole shows a predominance as the main metabolite circulating in serum of anastrozole-treated patients. It is pharmacologically inactive [17-19]. 
a)<smiles>C=C1CC2C(CCC3(C)C(=O)CCC23)C2(C)C=CC(=O)C=C12</smiles>

b)<smiles>CC(C)(C)c1cc(Cn2cncn2)cc(C(C)(C)C#N)c1</smiles>

Figure 4. Interaction of steroidal and nonsteroidal inhibitors with aromatase active centre - a) exemestane, b) anastrozole

The metabolism of phase II is based on coupling reactions catalyzed by specific transferases. In most cases, as a result of these reactions, a group is introduced into the xenobiotic molecule, which increases hydrophilicity, which in turn increases solubility in water. The resulting conjugates are then excreted in urine or bile. The purpose of the coupling reaction is to deactivate biological activity or to detoxify, so that the products of these reactions are in most cases biologically inactive. Coupling with active glucuronic acid (UDP) plays a special role in the metabolism of anastrozole. Because of the additional-OH hydroxyl groups in its structure, it is considered a relatively strong acid and a strongly hydrophilic compound. Its transfer to a specific substrate occurs after binding on the cell membrane with the UGT enzyme from the family of glucuronyltransferases (uridine 5'-diphospho-glucuronosyltransferase), which takes place mainly in the liver, kidneys and intestines. They are a family of enzymes whose detoxification function is based on catalyzing the reaction of glucuronic acid coupling with lipophilic substrate containing nucleophilic functional group in its structure. As in the case of cytochrome P450-dependent monooxidases, many UGT isoenzymes are distinguished. They differ in substrate specificity and expression specificity in specific tissues. Similarly to the P450 isoenzymes, they show differences in activity, which result from the induction of enzymatic protein genes. UGT enzymes are capable of glucuronidating many structurally diverse endogenous drugs and substrates and therefore play an important role in their elimination $[19,20]$.

Studies on anastrozole metabolism show that phase II of metabolism is mainly catalyzed by UGT1A4 isoenzyme. More- over, it was shown that this isoenzyme plays an important role in the direct glucuronidation of hydroxy-anastrozole formed as a result of oxidation in phase I. According to the results obtained, hydroxy-anastrozole was detected and quantified in plasma, mainly in conjugated form, as hydroxy-anastrozole O-glucuronide, and only to a small extent as non-conjugated form. This suggests that the hydroxylated metabolite is effectively conjugated by UGT enzymes. Anastrozole may also be directly glucuronidated to an anastrozole N-glucuronide, which is also catalyzed by UGT1A4. This reaction may occur on one of the atoms of nitrogen in the triazole ring, hence we are talking about N-glucuronidation. However, liver UGT1A4 is not the only enzyme involved in this reaction. UGT2B7 and UGT1A3 are also involved, but to a very small extent $[17,21]$.

To summarize the changes that anastrozole undergoes, Fig. 6 presents individual metabolic pathways including enzymes involved in them. Thanks to the conducted studies it is believed that hydroxylation is the main pathway of oxidation of phase I and $\mathrm{N}$-glucuronidation is the main pathway of drug conjugation in phase II. This data can serve as a basis for the design of clinical drug interactions and pharmacogenetic studies that consider metabolism with the P4503A4 and UGT1A4 enzymes and are designed to predict the clearance of anastrozole, i.e. the a volume of plasma from which drug is completely removed per unit of time.

\section{New therapeutic approaches to increase the effect of anastrozole}

In order to develop an appropriate anticancer therapy, it is important to understand the clinical pharmacology of the drug in question. Anastrozole has good bioavailability after oral 
<smiles>CC(C)(C#N)c1cc(Cn2cncn2)cc(C(C)(C)C#N)c1</smiles>

anastrozole<smiles>CC(C)(C)c1cc(O)cc(C(C)(C)C#N)c1</smiles>

hydroxy-anastrozole

Figure 5. Metabolism phase I of anastrozole

administration and is widely distributed in the bloodstream. It inhibits the concentration of estrone (E1) by $81-87 \%$, estradiol (E2) by $84-85 \%$ and estrone sulphate (E1S) by $94 \%$ [22]. It is intensively metabolized by liver microsomes with a half-life of 40-50 hours. It achieves maximum serum concentration within two to three hours after oral administration. The standard dose of anastrozole is $1 \mathrm{mg}$ per day, administered over a period of two to five years. This amount is optimal to inhibit the biosynthesis of estrogens. Studies in pharmacokinetics of anastrozole have shown that it does not affect liver or kidney dysfunction. Approximately $10 \%$ of the anastrozole dose is excreted unchanged in urine, while $60 \%$ is excreted in urine as metabolites present in urine $[16,23]$.

However, the use of anastrozole has many adverse side effects. These include disorders of the nervous system, musculoskeletal system, disorders of the stomach and intestines, connective tissue, metabolism, nutrition, vascular and general symptoms. Their occurrence is a consequence of low solubility of anastrozole in water, which quickly removes it from the bloodstream, resulting in short plasma half-life (on average 41-48 hours) and irregular drug intake [3].

\section{Nanotechnology}

Nanotechnology is a field of science that is often used to overcome the side effects of active pharmaceutical ingredients.

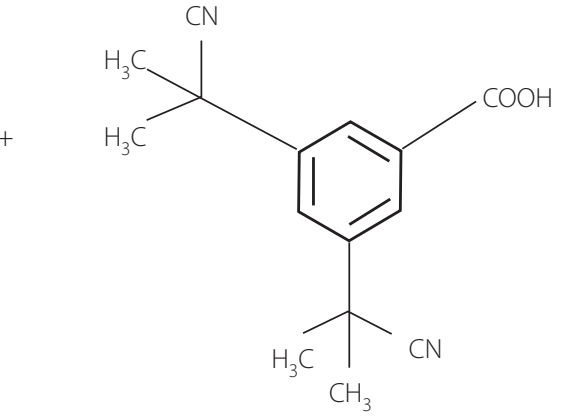

3,5-bis-(2-methylpropiononitrile)-benzoic acid
Particular role is played by nano-tools that provide a targeted approach to the administration of site-specific drugs through its coupling with carrier molecules such as nanocapsules, nanospheres and micelles [24]. Moreover, the trapping of hydrophobic compounds within specific nano-tools can significantly increase their solubility, delivery efficiency and, consequently, effectiveness. Referring to anastrozole, in 2006 Zidan et al. [25] published the first paper describing the incapsulation of this drug by using biodegradable microparticles based on lactic and glycolic acid copolymers (PLGA - poly(lactic-co-glycolic acid)). An extended anastrozole administration system was used in this case. One of the possible technological solutions used to increase the durability of drugs in the target place of their action is the application of therapeutic systems based on biodegradable polymers. Living matrices have the advantage of being biodegradable and gradually disappear, releasing the drug directly at the site of activity. Moreover, the administration of drugs in the form of microparticles facilitates injection [26].

PLGA has been extensively tested as a polymer carrier for biodegradable microspheres. PLGA microparticles have proven to be an effective means of delivering drugs belonging to various classes, including nonsteroidal anticancer drugs - which include anastrozole - as well as nonsteroidal anti-inflammatory drugs, peptides and steroid hormones. Choosing the right polymer composition with a known degradation 
<smiles>CC(C)(C#N)c1cc(C(=O)O)cc(C(C)(C)C#N)c1</smiles>

3,5-bis-(2-methylpropiononitrile)-benzoic acid<smiles>CC(C)(C)c1cc(Cn2c[n+](C(C)(C)C)cn2)cc(C(C)(C)C#N)c1</smiles>

anastrozole N-glucuronide<smiles>CC(C)(C)c1cc(O)cc(C(C)(C)C)c1</smiles>

Figure 6. Metabolic pathways of anastrozole including major enzymes involved in drug biotransformation

time allows it to be used in the production of a drug delivery system that releases the active ingredient at a predetermined rate. Both natural and synthetic polymers are used to prepare such microspheres. The most popular way to produce microspheres from PLGA - due to the process repeatability and homogeneity of particle size - is emulsification through solvent evaporation [25].

Formulated PLGA microspheres loaded with anastrozole showed that drug release profiles are characterized by two separate phases. The initial release phase occurs on the first day followed by a gradual second phase. The profiles of anastro- zole release from microparticles explain consecutive diffusion processes. The diffusion of aqueous, soluble solution into the matrix causes the drug to dissolve. Then it diffuses through the pores to a soluble medium [27]. Anastrozole in its structure does not contain any groups susceptible to ionization, therefore the change of $\mathrm{pH}$ does not affect its solubility [28]. Additionally, it has been shown that the drug release rate increases with increasing anastrozole load [25].

Another way of supplying anastrozole with nanotechnological tools is to use PLA-PEG-PLA nanoparticles, which are also biodegradable and non-toxic. They are synthesized from PEG 
(poly(ethylene oxide)), which is recognition-resistant by the immune system. In this case it was combined with PLA polylactide (poly(lactic acid)) [29]. This biocompatible system is capable of encapsulating anastrozole. In this case, a double emulsion technique was used, which allows effective delivery of anastrozole to the target cells. This system contributed to the prolonged release of the drug - up to 144 hours. This prolonged release is the result of affinity to the binding between the polymer and the drug and the polymer capacity to introduce the drug [30].

The three-block polymer used for the synthesis of nanoparticles has an amphiphilic character, which makes it suitable for incapsulating water-insoluble compounds, including anastrozole. Optimal size of nanoparticles is in the range of 10-200 $\mathrm{nm}$ - this allows direct targeting of tumors. Smaller particles can be removed and larger ones cannot enter the space of the vascular and interstitial tumor. Despite the increase in the level of proangiogenic signaling in cancer cells, which causes excessive vascularization of the tumor, delivery of the drug is possible because these vessels show increased permeability. Moreover, tumors are characterized by poor lymphatic drainage, which allows to retain macromolecules above 40 $\mathrm{kDa}$. Therefore, nanoparticles may use features of increased permeability and retention directed at solid tumors [31].

The efficacy of such a system was confirmed by cytotoxicity studies, which showed the ability of anastrozole-loaded nanoparticles to produce a similar cytotoxic effect compared to a free drug. The effectiveness of nanoparticles was also confirmed by the observation of expression of apoptotic genes like c-MYC, MAPK3 and MCL-1 in MCF-7 breast cancer cells. The MAPK3 gene belongs to the kinases activated by myogen, which are a fundamental component of the cellular signaling networkenabling the functioning of cells as an essential part of the organism. C-MYC protein is a transcription factor that plays an important role in the progression of breast cancer. It is a key regulator of cell cycle, cell proliferation and cell transformation. In case of access to survival factors, c-MYC deficiency induces apoptosis by activating its pathway through mitochondria and receptors. In turn MCL-1 gene is a proapoptotic member of the BCL-2 family of proteins, which show features of apoptosis inhibitors. The results of the study showed that all three genes were over-expressed, which leads to the induction of cancer cell apoptosis and thus directly confirms the efficacy of capsule drug delivery to the cells [30].

The use of modern nanotechnological techniques with the use of biodegradable polymers leads to prolonged release of some active ingredients. For anastrozole, further studies are needed to confirm the efficacy of drug-loaded nanoparticles and their suitability for clinical use. Any information available so far shall cover only in vitro studies involving cancer cell lines. Results of studies involving animals are also available [32].

\section{Transdermal drug release}

The most commonly used oral anastrozole preparation provides good therapeutic reactions. The maximum plasma concen- tration of the drug is maintained for approximately 2 hours and long-term systemic action with an average half-life of 40 to 50 hours. However, this is often associated with serious systemic adverse reactions. Patients suffering from nausea and vomiting are not eligible for oral medication. Transdermal Therapeutic Systems (TTS) are therefore a promising alternative and can help to avoid adverse side effects [33, 34]. They are a non-invasive and completely painless method by which a specified, fixed dose of the drug is delivered to the bloodstream through the skin. Their advantages include an even plasma concentration profile, sustained local release and, if necessary, the possibility of rapid elimination.

Oral therapy with aromatase inhibitors leads to uncontrolled delivery of a drug characterized by early, maximum drug concentration and worse bioavailability, and consequently increased number of side effects. On the other hand, low molecular weight, high partition coefficient and low melting point of anastrozole are favorable physicochemical properties, which may ensure its effective penetration through the stratum corneum of the epidermis.

The kinetics of anastrozole in the transdermal system was initially tested in vitro using Franz diffusion cells. The drug load, penetration and reinforcement in anastrozole transport were evaluated. The prolonged-activity self-adhesive anastrozole adhesive was then tested in vivo on beagle breed dogs. The patches were placed on the chest and abdomen skin of the animals. As expected, a lower initial transfer of anastrozole from experimental patches to systemic circulation was observed compared to oral drug administration in humans. However, in one day the level of anastrozole increased linearly and reached the maximum plasma concentration of 4.7 and $5.8 \mathrm{ng} / \mathrm{ml}$ at the time point Tmax $=24 \mathrm{~h}$. These values corresponded to $34 \%$ and $42 \%$ of the maximum concentration after one oral administration of $1 \mathrm{mg}$ of standard human dose of anastrozole [35].

In the case of anastrozole, the ability to control its release has many advantages, e.g. prolonged release leads to the desired prolonged duration of activity, while the concentration of the drug is sufficient to ensure adequate therapeutic activity. Moreover, it makes it possible to avoid an undesirable maximum concentration of the drug in the plasma. In addition, an easy-to-use system can potentially improve patient compliance [35].

\section{Summary}

The role of P450 cytochrome P450 in the metabolism of xenobiotics has been widely recognized and it is a molecular target for breast cancer chemotherapy. Aromatase catalyzing the biosynthesis of androgens into estrogens is particularly important in this enzymatic complex. It has been shown that inhibition of this reaction significantly reduces the level of estrogens in the bloodstream and thus limits the growth of tumors depending on the level of these hormones. One of the effective aromatase inhibitors is anastrozole, a nonsteroidal triazole derivative that 
inhibits P450 cytochrome 1A2, 2C8, 2C9, 3A4 isoenzymes involved in estrogen metabolism. As an exogenous substance, it is also metabolized in relation to enzymes of phase I and II, which leads to the formation of its metabolites: 3,5-bis-(2-methylpropiononitrile)-benzoic acid, anastrozole N-glucuronide, triazole and hydroxy-anastrozole O-glucuronide. The standard dose used in hormonal treatment of postmenopausal women is oral intake of $1 \mathrm{mg}$ of the drug daily. This amount is sufficient to achieve the desired therapeutic effect, which unfortunately also causes numerous side effects.

New methods of administration have been developed to reduce the incidence of anastrozole side effects and to increase plasma half-life. One of the solutions is the use of nano-tools that provide targeted delivery of the drug to increase the effectiveness of the therapy. For this purpose, the polymers used are PLGA, which are carriers of biodegradable micro-particles loaded with anastrozole. This system allows controlled release of the drug and thus affects the effectiveness of the therapy. It is also possible to use a completely non-invasive method of delivering the drug, i.e. administering it through the skin. This transdermal system allows for an even profile of plasma chemotherapeutic concentration based on a fixed, defined dose. Additionally, the physicochemical properties of anastrozole ensure its effective absorption by the epidermis.

The above aspects of anastrozole are the basis for a full understanding of both its mechanisms of action and the possibilities of designing new therapeutic approaches that increase the effectiveness of anticancer therapies.

\section{Acknowledgements}

The authors thank Prof. Zofia Mazerska for her knowledge and substantive support.

\section{Conflicts of interest: none reported}

\section{Izabela Piotrowska}

Gdańsk University of Technology

Faculty of Chemistry

Chair of Drug Technology and Biochemistry

ul. Narutowicza 11/12

80-233 Gdańsk, Poland

e-mail: izabela1.piotrowska@gmail.com

\section{Received: 27 Mar 2019}

Approved for printing: 6 May 2019

\author{
Abbreviations \\ ER - estrogen receptor \\ $\mathrm{Al}$ - aromatase inhibitor \\ COMT - catechol-O-methyltransferase \\ P450 19 - aromatase \\ E1 - estrone \\ E2 - estradiol \\ E1S - estrone sulphate \\ $\mathrm{NADP}^{+}$- nicotinamide adenine dinucleotide phosphate \\ $\log _{\mathrm{o} / \mathrm{w}}-$ partition coefficient \\ UDP - glucuronic acid \\ UGT - uridine 5'-diphospho-glucuronosyltransferase enzyme
}

PLGA - copolymer of lactic and glycolic acid

PEG - poly(ethylene oxide)

PLA - poly(lactic acid)

TTS - transdermal drug delivery system

\section{References}

1. Creighton CJ, Massarweh S, Huang S et al. Development of resistance to targeted therapies transforms the clinically associated molecular profile subtype of breast tumor xenografts. Cancer Res 2008; 68: 7493-7501.

2. Dębska S, Potemski P. Leczenie hormonalne chorych na raka piersi z nadekspresją receptora HER2. Onkol Prak Klin 2010; 6 (6): 301-310.

3. Buzdar AU. Anastrozole (Arimidex ${ }^{\mathrm{TM}}$ ) - an aromatase inhibitor for the adjuvant setting? Br J of Cancer 2001; 85: 6-10.

4. Buzdar AU, Jonat W, Howell A. Anastrozole versus megestrol acetate in the treatment of postmenopausal women with advanced breast carcinoma: results of a survival update based on a combined analysis of data from two mature phase III trials. Arimidex Study Group. Cancer 1998; 83: 1142-1152.

5. O'Shaughnessy J, Yasrdley DA, Burris HS et al. Randomized phase 3 trial of adjuvant letrozole versus anastrozole in postmenopausal patients with hormone receptor positive, node positive early breast cancer. Final efficacy and safety results of the femara versus anastrozole clinical evaluation (Face) trial. Thirty-Eighth Annual CTRC-AACR San Antonio Breast Cancer Symposium 2015

6. Bogacz A. Ocena wpływu wybranych roślin leczniczych na poziom transkrypcji genów CYP3A1 i CYP2D2 w badaniach In vitro. Projekt dyplomowy doktorski. UMP 2010.

7. Hiroshi S, Yoshitsugu S. Diversity and substrate specificity in the structures of steroidogenic cytochrome P450 enzyme. Biol. Pharm Bull 2012; 35: 818-823.

8. Lemke TL, Williams DA, Roche VF et al. Essentials of Foye's Principles of Medical Chemistry. USA 2006; 1303-1307.

9. Thomas MP, Potter BVL. The structural biology of oestrogen metabolism. Journal of Steroid Biochemistry \& Molecular Biology 2013; 137: 27-49.

10. Bańkowski E. Biochemia. Podręcznik dla studentów uczelni medycznych Edra Urban \& Partner 2016; 430-434.

11. Kowalewska-Łuczak I, Kmieć M, Terman A. Aromataza cytochromu P450 - kluczowy enzym syntezy estrogenów. Medycyna Wet 2006; 62 (8): 870-872.

12. Steinhilber D, Schubert-Zsilavecz M, Roth HJ. Chemia Medyczna. Wydawnictwo Medyczne i Farmaceutyczne MedPharm Polska. 2012; 510-512.

13. Berg JM, Tymoczko JL, Gatto GJ et al. Biochemistry. W.H. Freemanand and Company 2015; 792-795.

14. Grimm SW, Dyroff MC. Inhibition of human drug metabolizing cytochromes $\mathrm{p} 450$ by anastrozole, a potent and selective inhibitor of aromatase. Drug Metab Dispos. The American Society for Pharmacology and Experimental Therapeutics 1997; 598-601.

15. Product Monograph AstraZeneca Canada Inc. 2014

16. Bao T, Rudek MA. The clinical pharmacology of anastrozole. European Oncology \& Haematology 2011; 7: 106-108.

17. Kamdem LK, Liu Y, Stearns V et al. In vitro and in vivo oxidative metabolism and glucuronidation of anastrozole. Br J Clin Pharmacol 2010; 70: 854-869.

18. Lazaurus P, Sun D. Potential role of UGT pharmacogenetics in cancer treatment and prevention: focus on tamoxifen and aromatase inhibi-tors. Drug Metab Rev 2010; 42: 182-194.

19. Mutschler E, Geisslinger G, Kroemer HK et al. Farmakologia i toksykologia. Ed. 3. Wrocław: Wydawnictwo Medyczne i Farmaceutyczne MedPharm Polska 2013.

20. Mróz A, Mazerska Z. Glukuronidacja leków przeciwnowotworowych detoksyfikacja, mechanizm oporności czy sposób na formę proleku? Postepy Hig Med Dosw 2015; 69: 1462-1477.

21. Edavana VK, Dhakal IB, Williams S et al. Potential role of UGT1A4 promoter SNPs in anastrozole pharmacogenomics. Drug Metab Dispos 2013; 41: 870-877.

22. Geisler J, King N, Dowsett M et al. Influence of anastrozole (Arimidex), a selective, non-steroidal aromatase inhibitor, on in vivo aromatisation and plasma oestrogen levels in postmenopausal women with breast cancer. Br J Cancer 1996; 74: 1286-1991.

23. Yates RA, Dowsett M, Fisher GV et al. Arimidex (ZD1033): a selective, potent inhibitor of aromatase in postmenopausal female volunteers. Br J Cancer 1996; 73: 543-548.

24. Ediriwickrema A, Saltzman WM. Nanotherapy for cancer: targeting and multifunctionality in the future of cancer therapies. ACS Biomater Sci Eng 2015; 1: 64-78. 
25. Zidan AS, Sammour OA, Hammad MA et al. Formulation of anastro-zole microparticles as biodegradable anticancer drug carriers. AAPS Pharm Sci Tech 2006; 7: 61

26. Fernández-Carballido A, Herrero-Vanrell R, Molina-Martinez IT et al. Biodegradable ibuprofen-loaded PLGA microspheres for intraarticular administration: effect of labrafil addition on release in vitro. Int $J$ Pharm 2004; 279: 33Y41.

27. Freiberg S, Zhu XX. Polymer microspheres for controlled drug release. Int J Pharm 2004; 282: 1 Y18.

28. Arimidex ${ }^{\circledast}$ (Anastrozole) tablets prescribing information. 2002. Wilmington, DE: AstraZeneca. 2006.

29. Whitehead KA, Langer $R$, Anderson DG. Knocking down barriers: advances in siRNA delivery. Nat Rev Drug Discov. 2009; 8: 129-138.

30. Alyafee AY et al. Preparation of anastrozole loaded PEG-PLA nanoparticles: evaluation of apoptotic response of breast cancer cell lines. International Journal of Nanomedicine 2018; 13: 199-208.
31. Danhier F, Feron O, Preat V. To exploit the tumor microenvironment: passive and active tumor targeting of nanocarriers for anti-cancer drug delivery. J Control Release 2010; 148: 135-146.

32. Shavi GV, Reddy MS, Raghavendra R et al. PEGylated liposomes of anastrozole for long-term treatment of breast cancer: in vitro and in vivo evaluation. J Liposome Res 2016; 26: 28-46.

33. Amir E, Seruga B, Niraula S et al. Toxicity of adjuvant endocrine therapy in postmenopausal breast cancer patients: a systematic review and meta-analysis. J Nat/ Cancer Inst 2011; 103: 1299-1309.

34. Niravath P. Aromatase inhibitor-induced arthralgia: a review. Ann Oncol, 2013; 24: 1443-1449.

35. Regenthal R, Voskanian M, Baumann F et al. Pharmacokinetic evaluation of a transdermal anastrozole-in-adhesive formulation. Drug Design, Development and Therapy 2018; 12: 3653-3664. 